\title{
Event-related potentials evoked by passive visuospatial perception in rats and humans reveal common denominators in information processing
}

\author{
M. F. A. Hauser ${ }^{1,2} \cdot$ V. Wiescholleck ${ }^{1} \cdot$ J. Colitti-Klausnitzer ${ }^{1} \cdot$ C. Bellebaum ${ }^{3} \cdot$ Denise Manahan-Vaughan $^{1}$ (D)
}

Received: 14 December 2018 / Accepted: 25 February 2019 / Published online: 11 March 2019

(c) The Author(s) 2019

\begin{abstract}
In the human cortex, event-related potentials (ERPs) are triggered in response to sensory, cognitive or motor stimuli. Due to the inherent difficulties of conducting invasive mechanistic studies in human subjects, little is known as to the precise neurophysiological mechanisms that lead to their manifestation. By contrast, although much is known about synaptic and neural mechanisms that underlie information processing in rodents, very few studies have addressed to what extent ERPs are comparable in rodents and humans. Here, we explored this by triggering ERPs in both species during the passive observation of visuospatial imagery, shown in an oddball-like manner, using an experimental design that was equivalent. Several ERP-components were identified in the rodent cohort, corresponding, for example, to the human P1, N1, and P2. ERPs that are likely to reflect a rodent N2 and P300 were also detected. Deviance, as well as repetition effects were evident in both species, whereby rodent ERPs displayed more immediate response alterations to repeated stimuli and humans showed more gradual response shifts. These results indicate that humans and rodents may implement similar strategies for the passive perception and initial processing of visuospatial imagery, despite clear differences in their sensory and cognitive capacities.
\end{abstract}

Keywords Passive visual perception $\cdot$ Rodent $\cdot$ Human $\cdot$ Event-related potential $\cdot$ Spatial information $\cdot$ Electrophysiology

\section{Introduction}

The pathways supporting the perception of objects in humans and non-human primates have been the subject of many studies for almost half a century. The ventral stream, extending from V1 to V2 and V4, and the inferotemporal cortices (Ungerleider and Mishkin 1982; Mishkin et al. 1983; Felleman and Van Essen 1991) have been suggested to support the processing of objects in particular. Within area V1 of the primary visual cortex, neurons exhibit tuning towards basic visual features, such as luminance and spatial

Denise Manahan-Vaughan

denise.manahan-vaughan@rub.de

1 Department of Neurophysiology, Medical Faculty, Ruhr University Bochum, Universitaetsstr. 150, MA 4/150, 44780 Bochum, Germany

2 International Graduate School of Neuroscience, Bochum, Germany

3 Institute for Experimental Psychology, Heinrich-Heine University of Düsseldorf, Düsseldorf, Germany frequency, that are then combined along V2 and V4 to generate increasingly complex representations (Gallant et al. 1993; Ito and Komatsu 2004). Finally, in the inferotemporal areas, complex object categories, such as body parts, faces, and inanimate objects are represented (Perrett et al. 1982). Along this pathway, neurons thus show responses to increasingly complex feature combinations, while at the same time also showing increasing receptive field sizes (Gross et al. 1969).

Although much of our knowledge with regard to object perception comes from primate research, a recent increase in interest in rodent object perception now sets the stage to consolidate findings from mechanistic studies that feature rats and mice as the dominant species, with studies on higher cognitive functions conducted in humans and non-human primates. Studying the underlying neuronal response properties, several studies have shown that along the trajectory from V1, through latero-medial, latero-intermediate, latero-lateral, and occipito-temporal areas, an increasing tolerance to position and viewpoint is observed in the awake rat, similar to what is seen in primates (DiCarlo et al. 2012; Vermaercke et al. 2014; 
Tafazoli et al. 2017). Furthermore, it has been shown that $\mathrm{V} 1$ displays the shortest response latencies at around $40-50 \mathrm{~ms}$, closely followed by latero-medial, and -intermediate areas at around $50 \mathrm{~ms}$, after which latero-lateral and occipito-temporal areas respond at roughly $80 \mathrm{~ms}$ (Vermaercke et al. 2014). However, while neurons in the rat primary visual cortex exhibit tuning similar to that observed in primate $\mathrm{V} 1$, they lack functional columns (Ohki et al. 2005). Despite this difference, it was reported that, when rodents process multiple stimuli, neurons along the axis of tested regions become sharply energyindependent, meaning that luminance of presented objects had less of an impact in higher order areas (Tafazoli et al. 2017). The same study demonstrated that neurons in higher visual areas become increasingly view-invariant to objects. These findings suggest, that at least on a singleunit level, a similar progression of information processing along the visual areas occurs in rats, humans and nonhuman primates. To what extent the temporal dynamics of cortex-wide responses are comparable between rodents and primates is, however, not clear.

In this study, we set about to explore to what extent passively viewed visuospatial information is processed in equivalent ways by the rodent and human cortex. To do this, we created a test paradigm that was designed to investigate cortical novelty responses in the context of object processing by means of event-related potentials (ERPs). We observed that humans and rodents appear to utilize similar strategies for the passive perception and initial processing of visuospatial imagery.

\section{Experimental procedures}

To compare evoked potentials in humans and rodents, we used a passive visuospatial oddball-like paradigm, where both species were presented with novel abstract complex objects in a repeated manner (standards), interleaved with the same objects in a configurationally changed variant (deviants; Fig. 1). In contrast to the classical oddball paradigm, where standards are repeated throughout the experiment, we changed the standard objects every 17-21 repetitions. The animals were shown these objects via two monitors while they were drinking from a juice dispenser (Kemp and Manahan-Vaughan 2012), during which we recorded EEG signals from 12 cranially implanted electrodes spanning from the frontal to occipital cortices. Our human subjects were exposed to the same paradigm whilst participating in a distractor task, to ensure they were looking at the objects, but not actively attending them.

\section{Rodent study}

The rodent study was carried out in accordance with the European Communities Council Directive of 22 September 2010 (2010/63/EU) for care of laboratory animals. All experiments were performed according to the guidelines of the German Animal Protection Law and were approved by the North Rhine-Westphalia State Authority (Landesamt für Naturschutz, Umweltschutz und Verbraucherschutz, Nordrhein Westfalen, Germany). All efforts were made to reduce the number of animals used.

The animal cohort consisted of 13 adult male hooded Lister rats (Charles River Breeding Laboratories, Sulzfeld,

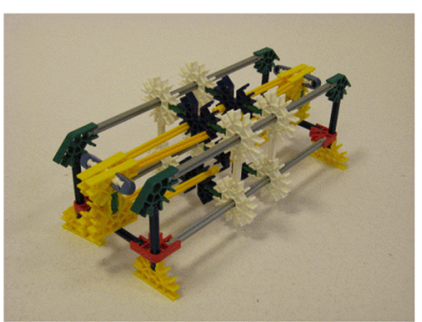

Standard

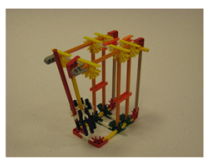

$600 \mathrm{~ms}$

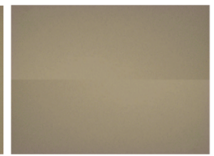

400-700ms

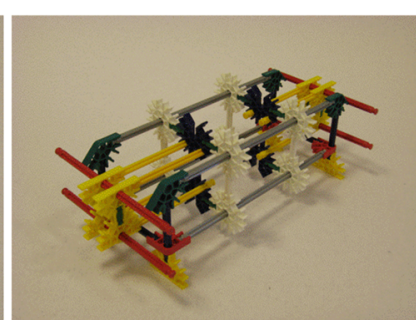

Deviant

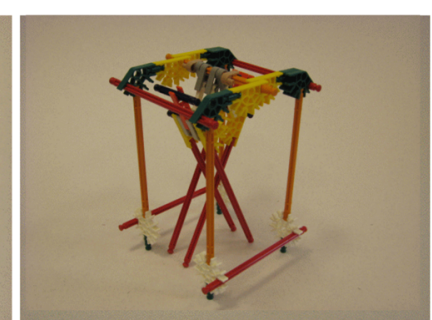

Standard

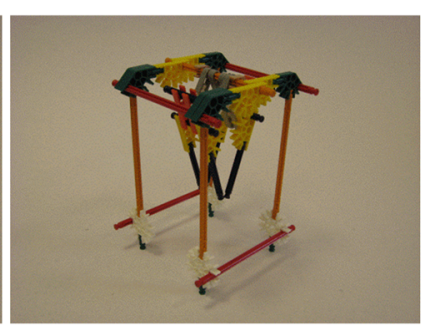

Deviant

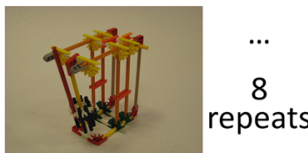

$600 \mathrm{~ms}$

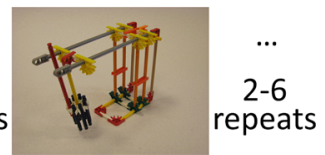

$600 \mathrm{~ms}$

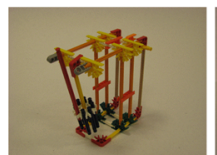

$600 \mathrm{~ms}$

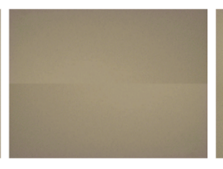

$400-700 \mathrm{~ms}$

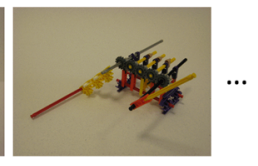

$600 \mathrm{~ms}$

\section{$S_{1}^{1} S_{2}^{1} S_{3}^{1} S_{4}^{1} S_{5}^{1} S_{6}^{1} S_{7}^{1} S_{8}^{1} S_{9}^{1} S_{10}^{1} S_{11}^{1} D^{1} S_{13}^{1} S_{14}^{1} S_{15}^{1} S_{16}^{1} S_{17}^{1} S_{1}^{2} S_{2}^{2} S_{3}^{2} S_{4}^{2} S_{5}^{2} S_{6}^{2} S_{7}^{2} S_{8}^{2} S_{9}^{2} S_{10}^{2} D^{2} S_{11}^{2} S_{12}^{2}$}

Fig. 1 Stimuli, time-line and trial order. Top row: examples of the stimuli used. Middle row: timing of an example trial for the rodents. Bottom row: ordering of standards and deviants for two objects. The superscript denotes objects being shown as standard (S) or deviant (D). The subscript denotes presentation number. Bold letters indicate which epochs were later extracted for analysis 
Germany). At the time of surgery they were 7-9 weeks old. The animals were housed separately on a $12 \mathrm{~h}$ light/dark cycle, and under controlled temperature $\left(22 \pm 2{ }^{\circ} \mathrm{C}\right)$ and humidity $(55 \pm 5 \%)$. Both food and water were provided ad libitum.

\section{Electrode placement}

To be able to differentiate ERP-components according to their cortex-related topographical properties, 13 stainless steel screws (with $0.8 \mathrm{~mm}$ diameter, a length of $1.4 \mathrm{~mm}$, and an impedance of $<1 \Omega$ ) were implanted into the animal's skull to enable recording of cortical EEG. Each screw (Optotec, Rathenow, Germany) was attached to silver wire (Axona, St. Albans, UK). Twelve screws served as recording electrodes and one was used as a ground electrode. Screw insertion was done while the animal was deeply anesthetized using sodium pentobarbital (Narcoren, Merial GmbH, Germany, $52 \mathrm{mg} / \mathrm{kg}$, intraperitoneally). The animal was placed in a stereotactic unit. Following application of the local anesthetic, lidocaine, a scalp incision of ca. $3 \mathrm{~cm}$ in length was made from a point between the eyes, along the midline towards the back of the skull and the periosteum was then removed. Small drill holes were then made in the skull to correspond to the electrode positions. The drill holes were positioned in five rows that were aligned $3 \mathrm{~mm}$ on either side of the midline (see: Fig. 6h). Two rows were positioned $1.5 \mathrm{~mm}$ and $4.7 \mathrm{~mm}$ anterior to bregma. At these anterior positions two further electrodes were placed exactly on the midline. Three rows of two electrodes were placed $1.9 \mathrm{~mm}$, $4.16 \mathrm{~mm}$, and $6.9 \mathrm{~mm}$ posterior to bregma. The ground electrode was placed in the skull over the cerebellum. This arrangement of electrodes allowed covering the largest portion of the rodent cortex, while at the same time allowing some degree of specificity to different functional areas. The electrode screws were inserted without piercing the dura. After placement the screws were then attached via the silver wire to a 16-channel Headstage (Axona, St. Albans, UK). The connections were covered and the assembly was attached to the skull using dental acrylic (Paladur, Heraeus Kulzer, Wehrheim, Germany). Pre-and postoperative analgesia was implemented using Meloxicam (Boehringer Ingelheim Vetmedica GmbH, Ingelheim, Germany). At least 10 days were allowed to elapse after surgery before apparatus training and experiments were commenced.

The most anterior row of electrodes covered the frontal association cortices (FrA and FrAz) (see: Fig. 6h). The second row of anterior electrodes covered the primary motor cortex (M1) laterally, and on the midline the cingulate cortex area $1(\mathrm{Cg} 1)$. The third row (immediately posterior to bregma) covered the primary somatosensory areas (S1) (see: Fig. 6). The fourth row covered the parietal association

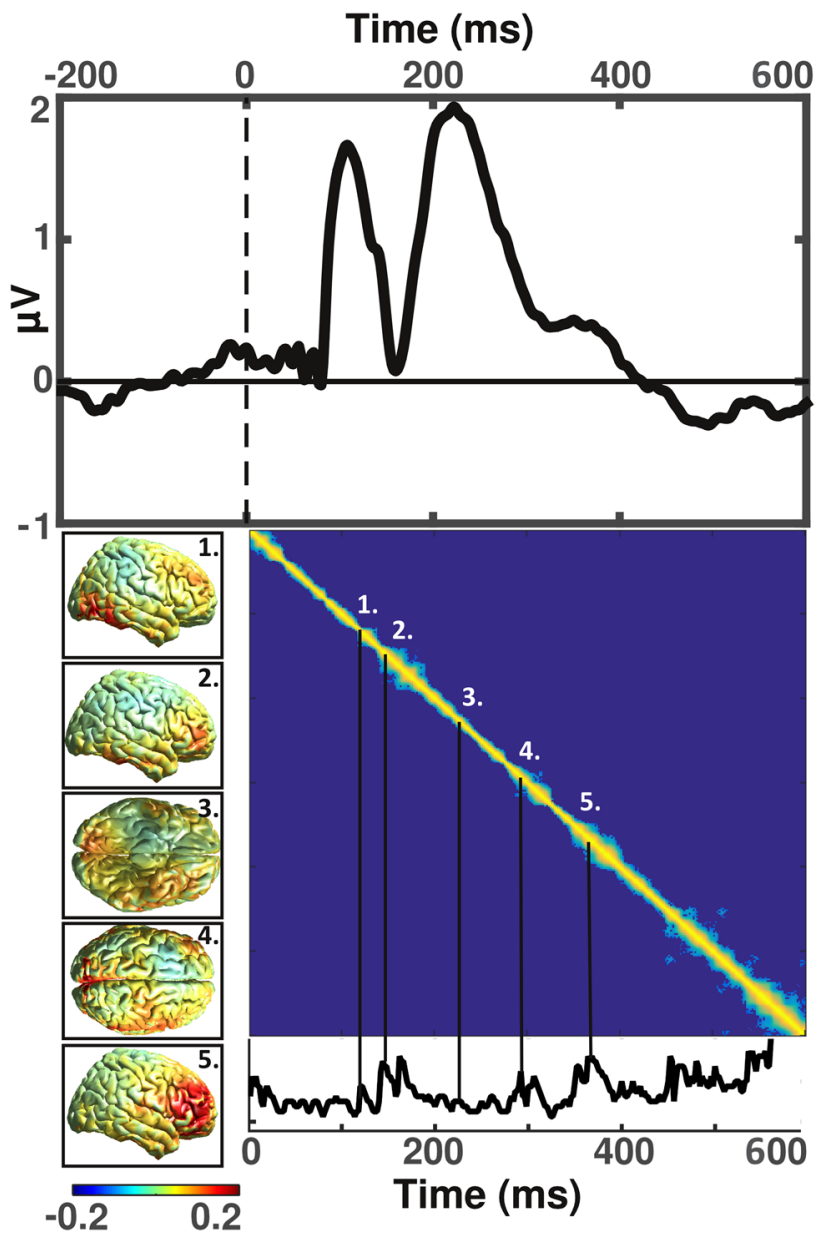

Fig. 2 Event-related potential response and corresponding sources of human subjects. Top: human grand average event-related potential (ERP) derived from parietal electrodes. Bottom right: representational similarity matrix calculated for the time span of 0-600 ms and 'thresholded' at $p<0.05$, that includes a time series indicating the time points at which source patterns correlated significantly. Bottom left: source estimates for time windows of interest corresponding to the numbering included in the bottom right panel

cortices (PtA), whilst the last row was situated above the primary visual cortex (V1M).

\section{Recording apparatus}

To ensure that the rodents viewed the stimuli presented, we implemented a similar approach as already described in a previous study (Kemp and Manahan-Vaughan 2012): animals were placed in a recording chamber made of gray Perspex that contained a triangular annex where, through a hole ( $6 \mathrm{~cm}$ above ground), the animals could drink from a nozzle of a drinking bottle filled with carrot juice mixed with honey. The two flanks of this triangle-shaped part of the box contained removable windows made of non-reflective clear Perspex. Two monitors were placed behind these windows, 
one on each side, at a distance of $40 \mathrm{~cm}$. Apart from the roof of the box, which was open to allow free movement of the cable connecting the headstage to the amplifier, the outsides and lower side of the box were covered in tin foil, which was grounded via a cable attached to the input connection of an electrophysiological recording system (Axona, St. Albans, UK).

To familiarize them with the recording procedure, the animals were handled individually for $10 \mathrm{~min}$ per day for at least a week before the start of the experiment. A key element of the experimental design is that the animals choose a position at the windows of the recording chambers so that they can view both computer monitors simultaneously. To achieve this, during the experiment they were able to drink carrot juice in this position. For at least 3 days before the experiment, the animals spent around 30 min per day in the recording chamber, during which they were able to drink the juice ad libitum. During this time, the monitors, on which images were later displayed, were turned on, to habituate the animals to the level of luminance coming from the screens.

\section{Stimuli and experimental trials}

A randomized passive visuospatial oddball-like paradigm was designed to investigate the effects of stimulus repetition, as well as spatial change, on ERP-components. The stimuli used in this set-up were the same as those employed by Bellebaum et al. (2013; Rüther et al. 2014a, b; Ghio et al. 2016) (Fig. 1). These images consisted of photos of 15 abstract objects that were constructed from toy construction parts $\left(\mathrm{K}^{\prime} \mathrm{NEX}^{\mathrm{TM}}\right)$. These stimuli were selected for the present study, as they had been created and standardized such that they were complex and real, yet provided little to no association with any meaningful objects, and, therefore, eliminated any affordance humans might perceive in them. This effectively allowed presenting our human subjects with images that were as novel to them as they were to the rodent cohort. The objects were photographed so that the threedimensional configuration of the structure was easily visible (Fig. 1). For each object, two corresponding deviants were constructed, in which the standard object was slightly altered. These alterations consisted of replacing a certain component of the object, effectively changing its spatial configuration (Fig. 1). The sequence of events in one particular experimental trial of the experiment was as follows: for both, humans and rodents, one standard stimulus was repeatedly shown between 17 and 21 times, after which a new standard was introduced. For one of these repetitions the deviant was shown instead of the standard, randomly from the 11th to the 15 th repetition. The initial presentation of a standard, and its corresponding ERP is what we here refer to as the novel object, and the corresponding novelty response, whilst the configurational change in the deviant stimulus elicited the deviant response.

\section{Procedure}

For the experiment, the animals were placed in the recording chamber. Throughout the experiment, the room lights were turned off. Each image was presented for $600 \mathrm{~ms}$, with a random inter-stimulus interval between 400 and $700 \mathrm{~ms}$. This was held constant throughout each entire experimental block, thus no planned breaks or different intervals were implemented when a new standard, or the deviant was shown. Importantly, images were only presented while the animals were drinking to ensure that their heads were correctly placed to view both computer screens. Whenever they stopped, the presentation was paused manually by the experimenter, and only restarted after the animals resumed drinking. If at any point the animals stopped drinking for more than $5 \mathrm{~s}$, all events up until the presentation of the next object were discarded from analysis. The rodent experiment was carried out over at least 4 days. On each day, the experiment was further divided into four sessions. Each session consisted of six trials in which the stimuli were presented as described above. Between the sessions, the drinking bottle was removed for around 3-5 min. In five animals, data acquisition was extended beyond four days (for three animals this meant a total of 5 days, for two animals 6 days), because the animals did not want to drink for more than $30 \mathrm{~min}$ in one session, so that the experiment was aborted.

\section{Data acquisition and preprocessing}

During the recording sessions, aheadstage, serving as an AC-coupled unity-gain operational amplifier, was plugged onto the 16-channel connector, from where the signal was passed through the Axona data-acquisition system where it was amplified to be passed on to the recording computer. Finally, the continuous EEG data were exported to Matlab at a sampling rate of $250 \mathrm{~Hz}$ for further analysis. There, using the EEGLAB toolbox (Delorme and Makeig 2004), the data from each recording session were filtered using a high- and low-pass cut-off frequency of 0.5 and $70 \mathrm{~Hz}$, respectively. After this, the individual channels were assessed and, if consistently noisy as evidenced by clear and broad departure from other electrodes in its power spectrum, removed from further analysis. In two animals, this was the case for the right frontal electrode, where the left counterpart was used to replace the missing electrode. The left somatosensory channel contained too many artifacts in eight of the 13 animals, thus, we chose to exclude it entirely from further analysis. As one of our main aims, next to exploring visuospatial deviance, was to investigate the effects of stimulus repetition on the processing of visual information in our two 
populations, we analyzed the first ten repetitions of every presented standard. These ten repetitions were the minimum number of presentations of the standard before a deviant could potentially occur. For these conditions of interest, the data were segmented into epochs of $1200 \mathrm{~ms}$, starting from $200 \mathrm{~ms}$ before the presentation of a given image. The data were re-referenced to the average of all electrodes, after which, by means of visual inspection, noisy and unusable trials were removed. Specifically, we excluded epochs where the signal was partially clipped or lost, due to the juice coming into contact with the surrounding aluminum foil and the rodent at the same time. Independent component analysis, as implemented in the EEGLAB toolbox was used to decompose the data into as many temporally uncorrelated components as there were electrodes. This allowed subtracting noise and artifacts that were generated by the drinking motion, while having a relatively small impact on the signal of interest. In most cases this was just a single component, identified through being represented in the data during the baseline period, as well as the time after stimulus onset, and being equally prominent in all electrodes. Care was taken to minimize the impact this subtraction had on component amplitudes, while resulting in the smoothest possible baseline. Finally, after baseline subtraction, all epochs belonging to a particular condition were averaged per channel.

\section{Human study}

A total of 30 healthy, medication-free, adult participants with normal or corrected-to-normal vision participated in this study, which received prior approval from the Ethics Committee of the medical faculty of the Ruhr University Bochum. After excluding three individuals due to too many trials $(>50 \%)$ being rejected by the automatic artifact rejection algorithms (see "Data acquisition and preprocessing" section), the final sample consisted of 16 females and 11 males, with a mean age of 30.5 years.

\section{Procedure}

The participants were seated between 70 and $90 \mathrm{~cm}$ from the screen. The stimulation in each individual trial of the task used in human participants was the same as for the rodents (see above). To ensure that the human participants were only passively processing the objects, they were given a distractor task, which required the visual discrimination of a small square that appeared in the centre of the screen after the onset of the object picture. Participants responded with a right-or left-handed button press, depending on whether the square was white or black. On-screen instructions explained the task, and that they were to respond as fast as possible. Before the actual experiment started, a short training trial was presented. Participants were presented the images for at least 600 and maximally $800 \mathrm{~ms}$ before the target square appeared, lasting until a response was made, or until a total of $1200 \mathrm{~ms}$ since the appearance of the image had passed. As was the case for the rodents, the inter-stimulus interval was between 400 and $700 \mathrm{~ms}$. Furthermore, the experiment consisted of four blocks, with each block consisting of 15 trials (one per object), and their respective deviants. The deviants alternated from one block to the next. Between these blocks, participants were able to take a break.

\section{Electrode placement}

Using the ActiCap system (Brain Products GmbH, Munich, Germany), the participants were fitted with a $64-\mathrm{Ag} / \mathrm{AgCl}-$ electrode cap, which was secured with a chin-strap and configured according to the $10 \%$ extension of the international 10-20 system. All electrodes were referenced to $\mathrm{FCz}$, with the ground located at AFz.

\section{Data acquisition and preprocessing}

Before the recording, the electrodes were filled with electrode gel, and impedance was kept below $5 \mathrm{k} \Omega$. The signal was digitized at $500 \mathrm{~Hz}$. Using BrainVision Analyzer (BrainProducts $\mathrm{GmbH}$, Munich, Germany), the data were 'cleaned' using independent component analysis to identify eye-blink artifacts, and subjected to a bandpass filter using 0.5 and $70 \mathrm{~Hz}$ as low and high cut-off frequencies, respectively. After that, as in the rodent preprocessing pipeline, the data were segmented into epochs of $1200 \mathrm{~ms}$ length, from $200 \mathrm{~ms}$ before to $1 \mathrm{~s}$ after an event of interest. Automatic artifact detection, with a maximum voltage step of $50 \mu \mathrm{V}$, and an epoch-wide maximal and minimal amplitude difference of 150 and $0.5 \mu \mathrm{V}$, respectively, was then applied to remove trials with artifacts.

\section{Analysis}

\section{Source analysis}

For both cohorts, we first performed a source reconstruction of the recorded ERPs over time, to allow identification of the different processing stages. Note that for the humans' source reconstruction we used a subset of 14 individuals that showed the strongest source power in the occipital cortex during the $\mathrm{P} 1$ component (defined as greater than a $5 \%$ signal increase relative to the baseline). The source estimation was done using the Matlab-toolbox fieldtrip (Oostenveld et al. 2011). For the rat cohort, the forward model was built based on a rodent T1-scan published by Valdés-Hernández et al. (2011). Specifically, we created 
a mesh of the combined gray and white matter estimates, upon which we added a thin layer of a second mesh representing extracerebral fluid. We chose not to distinguish between white and gray matter in the forward model, since it was difficult to meet the requirement of the meshes being non-intersecting and closed, and because we were interested mostly in superior cortical, thus gray matter areas, that we deemed more likely to be estimated correctly with the low spatial resolution provided with our electrode number. A head model was then created using the openmeeg-software (Kybic et al. 2005; Gramfort et al. 2010). Volume conduction for the gray and fluid volumes was set to 0.33 and 1 , respectively, and the two layers consisted of 3998 and 1998 vertices, respectively. The leadfield matrix was created based on this head model and on ten electrodes (excluding both electrodes above the somatosensory cortices), as well as a rectangular 29 by 28 by 7 source grid of 5684 nodes spaced $0.5 \mathrm{~mm}$ apart, spread across the neocortical region. Again, we chose to focus on the superior cortical areas due to our two-dimensional electrode placement, thus only created a depth of $7(3.5 \mathrm{~mm})$ layers. For the human cohort, we used the boundary element head model provided as a part of the fieldtrip toolbox, which consists of three layers, being scalp, skull, and brain. The human source-grid consisted of 15 by 18 by 15 sources spaced $1 \mathrm{~cm}$ apart.

We used linear constrained minimum variance, a beamformer scanning method, where each potential dipole is estimated separately. This method constructs a spatial filter to separate one source from the next, by creating the filter for each source such that when it is applied to another source, the resulting variance is as close to zero as possible. This is done using a covariance matrix derived from the data in question, which here were all epochs belonging to first and tenth presentations. We calculated the filter based on all time points in these epochs, thus from - 200 to $1000 \mathrm{~ms}$. We used the tenth presentation as a baseline, to which the novelty response was compared. Subsequently, we used the filter to estimate the source power of the novelty response relative to the baseline across $40 \mathrm{~ms}$ time windows, centered on each time point starting from stimulus onset. Source power of the novelty response was calculated at each time point by subtracting the estimate for the baseline from the estimate for new objects, and dividing it again, by the estimate for the baseline (the percentage-wise difference).

\section{Multivariate analysis of source estimates}

One important question was to what extent the human, and especially the rodent, source estimates could be used to identify different processing stages, that in turn could corroborate the choice of ERP-components to analyze. For this, we correlated the brain patterns elicited at every time point to all other time points. This yielded a matrix of $R$ values indicating to what extent source estimates were coherent over time. Naturally, and in part due to the calculation of source estimates spanning over time windows of 40 and $80 \mathrm{~ms}$ in the rat and the human cohorts, time points adjacent to one another produced larger correlations than those further apart in time. However, we found some areas along this temporal axis produced broader correlations than others, which were indicative of less transient patterns, that may constitute a "processing stage". To quantify this statistically, we employed permutation statistics, whereby we randomly permuted the source estimates along time points and correlated this with the original, non-permuted data a total of 1000 times. This effectively created a distribution of what we could expect if the time points were interchangeable (the null-hypothesis distribution). We then 'thresholded' the original matrix according to how many of the correlations with permuted data yielded stronger $R$ values than the original. We selected a threshold of 50 , corresponding to a $p$ value of 0.05 . To quantify different cortical states that contributed to the novelty response, and to relate these to the different components, we calculated a time series that indicated how stable the source estimates for a given time point were over time. This was done by counting the number of consecutive time points in which source estimates were significantly correlated, following each respective time point. Here, we allowed the 'skipping' of a single non-significant time point, if it was followed by further significant correlations. The resultant time series indicated different peaks, which coincided with many of the ERP-components for both rats and humans.

\section{Analysis of repetition and deviant effects}

After identifying the different components of interest, we analyzed the effects of repetition and deviant effects separately in the electrodes in which we found the respective components to be the most prominent. Further analyses were done for each component using the subject-wise mean peak amplitude, for each of the first ten presentations, as well as the deviant. This amplitude was, in most cases (the exception being the human $\mathrm{N} 2$, and the rodent $\mathrm{P} 3$ ), the maximum/minimum of the condition mean in a time window of $\pm 30 \mathrm{~ms}$ for the humans, and $\pm 32 \mathrm{~ms}$ for the rodents, from the respective peak of the grand average component. This was chosen to account for subject-wise variability in component latency. To better control for type I errors, we averaged the last six repetitions into two conditions, consisting of repetitions 5-7, and $8-10$ that we refer to as the intermediate, and late repetition phase trials, respectively. The repetition effects were tested using a one-way repeated measures analysis of variance (ANOVA) with the single factor of repetition number constituting six levels, from presentations one to four, as well as intermediate and late repetition phases. 
If found to be significant, we then tested three functions in terms of their respective fit to each subject's mean amplitudes over all ten repetitions. The first was a basic linear function, the second a quadratic, and the third a cubic polynomial. For these curves we evaluated the adjusted $R^{2}$, which indicates the amount of variance explained by a curve, while penalizing (dividing by) the number of parameters. This was done separately in each subject, and the resultant $R^{2}$ adj from the linear model was compared to both quadratic and cubic functions were compared in a Wilcoxon signed rank test to test whether the non-linear functions were significantly better at explaining the data. In cases where both, quadratic and cubic functions, were better at explaining the repetition effect, these were further contrasted. In the case of nonsignificance, the repetition effect was assumed to follow the more parsimonious model.

Finally, we conducted a separate analysis to investigate whether the deviant image was significantly different from the late repetition phase, using a paired $t$ test, with $\alpha=0.01$ to account for multiple comparisons in each of the cohorts. For the rodent P3, there was no clearly discernible peak in the grand average, for which we, therefore, chose the condition-specific peak in a $300 \mathrm{~ms}$ time window, from 200 to $500 \mathrm{~ms}$

\section{Results}

\section{Human novelty response progresses from V1 to inferior temporal cortex, and posterior midline}

Closer scrutiny of the representational sample of our human cohort, on which we ran the source analysis, revealed a progression from visual cortices (Fig. 2, inset '1.') to inferotemporal areas (Fig. 2, inset '2.'), and then a distributed activity pattern spanning temporal, parietal, and inferior occipital areas (Fig. 2, inset '3.') that occurred in response to the presentation of novel visuospatial items (Fig. 1). Subsequently, the posterior midline region was found to be maximal (Fig. 2, inset '4.'), indicating a generation in the posterior cingulate. Finally, frontal activity was strongest (Fig. 2, inset '5.'). Particularly the occipital (at $120 \mathrm{~ms}$ ), inferotemporal $(144 \mathrm{~ms})$, posterior medial $(292 \mathrm{~ms})$ and frontal $(352 \mathrm{~ms})$ increases in source power, coincided with an increase in temporal stability over time (Fig. 2, bottom).

\section{Human ERP-components reflect the different stages of the novelty response}

Examination of the ERPs generated by the visual stimuli (Fig. 3a-n) identified different stages corresponding to different ERP-components (see top of Fig. 2, for parietal example), such as the P1 for primary visual areas, and the
$\mathrm{N} 1$ for inferotemporal source power. The P2 corresponded to the parietal power increase during its upward slope, and the posterior cingulate activity during its descending phase, although this component appeared to be less characterized by any particular source estimation pattern. Furthermore, in this downward slope, we found that the novelty ERPs displayed a markedly more negative amplitude relative to repeated objects (Fig. 4). We hypothesized this to be the N2, and thus considered this an individual component that we then investigated based on each conditions' difference amplitude to the tenth presentation. This yielded what indeed appeared to be a component largely specific to the first presentation of a new item, with an amplitude of $-3.41 \mu \mathrm{V}$ (SD 3.06 ), relative to the late repetition phase. The late, mostly frontally distributed, increase in source power corresponded temporally to the P300. The details of which electrodes and time windows we used for each component in the following analyses can be found in Table 1 .

\section{Rodent novelty response passes from $\mathrm{V} 1$ to parietal, lateral visual, and retrosplenial areas}

In the rodents, we found an initial activation of visual cortices at around 32-40 ms (Fig. 5, inset '1.'). This corresponds to the neuronal response latency in the primary visual cortex (Vermaercke et al. 2014). This then progressed to parietal areas starting from $52 \mathrm{~ms}$ (Fig. 5, inset '2.'), from where the novelty response simultaneously extended towards occipitotemporal areas at $120 \mathrm{~ms}$ (Fig. 5, inset '3.'), as well as to the retrosplenial cortex, and at $152 \mathrm{~ms}$ to the anterior cingulate region (Fig. 5, inset '4.'). Despite the brevity and short latencies of the early rodent ERP-components and the corresponding stages, the rodent brain patterns also displayed several peaks in temporal coherence, suggesting that the localization to the visual cortex appears to be temporally separable from the subsequent increase in parietal estimates (Fig. 5). The subsequent spread to occipito-temporal, retrosplenial, and finally also to anterior cingulate areas appeared to form a complex. We also identified a late temporal coherence in brain patterns, between 300 and $400 \mathrm{~ms}$, which did not correspond to any particular cortical increases or decreases in source power (and hence formed its own discrete stage). Given that, at the electrode level, the novelty response was also prominent at this time point (see below), the lack of source differences may have arisen from a larger amount of variance over time in rodents, or be indicative of a subcortical generation mechanism.

\section{Rodent ERP-components reflect discrete stages of novelty processing}

The rodent ERPs (Fig. 6a-j) displayed a similar progression as that observed for the human potentials, in that a small 

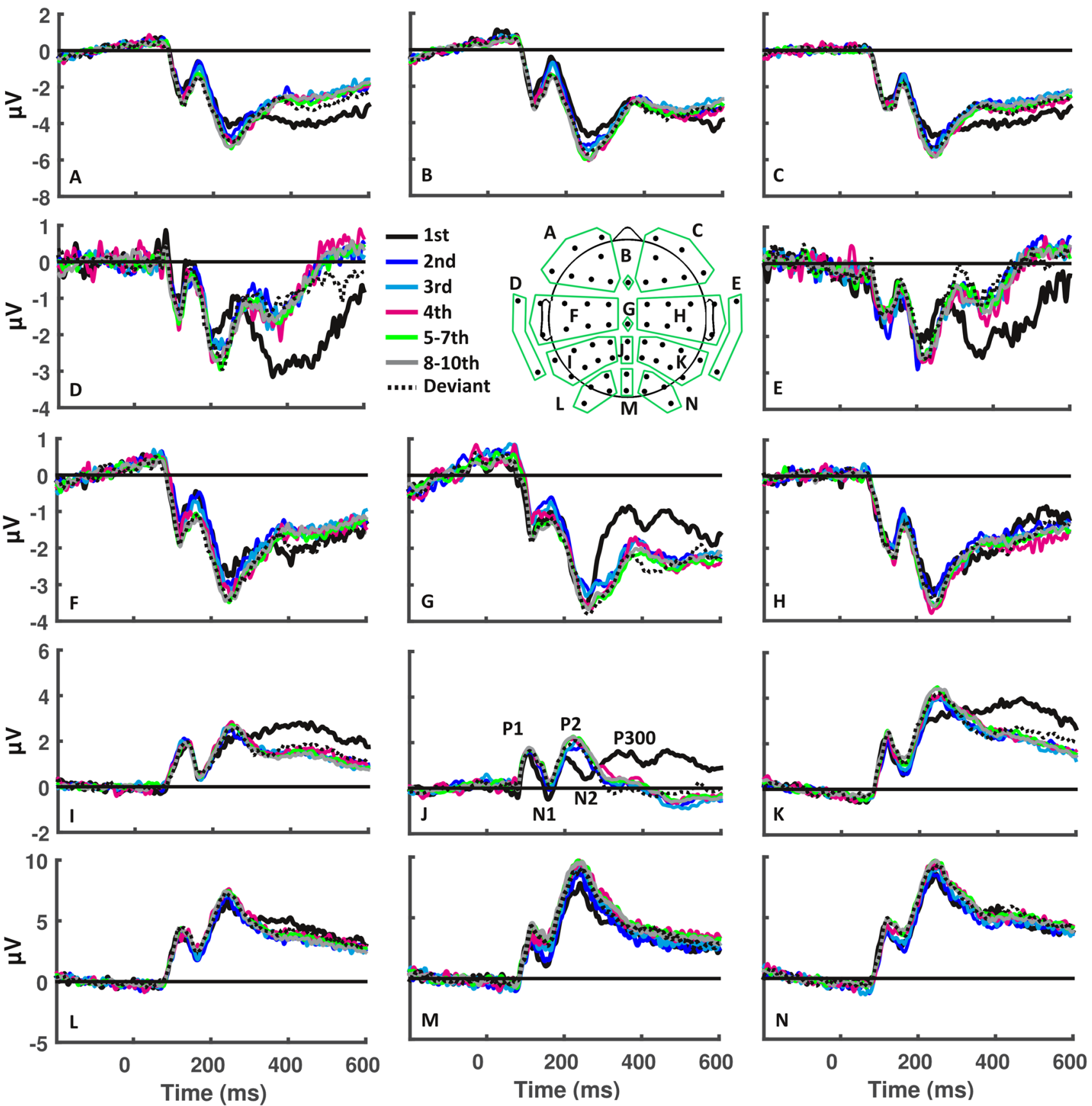

Fig. 3 Human event-related potentials elicited by visuospatial information processing. Event-related potentials (ERPs) were recorded across 14 subsets of electrodes, the locations and constellations of which are indicated by the letters in the schema of the electrode lay-

positivity at $40 \mathrm{~ms}$ (P40) was observed in occipital electrodes. This corresponded from a temporal point of view to the stage indicating the increase in primary visual cortex source estimates. Following this, we found a negativity at $80 \mathrm{~ms}$ (N80), that was most prominent in occipital electrodes (Fig. 6e, j), and which corresponded to the second stage we identified in the parietal source power increase. Following out. ERPs shown here correspond to responses evoked by the first four repetitions, as well as the averaged responses that occurred in the intermediate and late repetition phases. Specific ERP-components are indicated for the middle parietal electrodes $(\mathrm{J})$

the $\mathrm{N} 80$, we found a positive deflection, again in occipital electrodes, at roughly $135 \mathrm{~ms}$ (P135; Fig. 6e, j). During its descending phase this deflection overlapped with a negativity at $160 \mathrm{~ms}(\mathrm{~N} 160$; Fig. $6 \mathrm{~g}, \mathrm{j})$ that was very pronounced in the second middle electrode, and was temporally in line with anterior cingulate activity. Together, the P135 and the N160 corresponded to the rapid distribution from parietal 

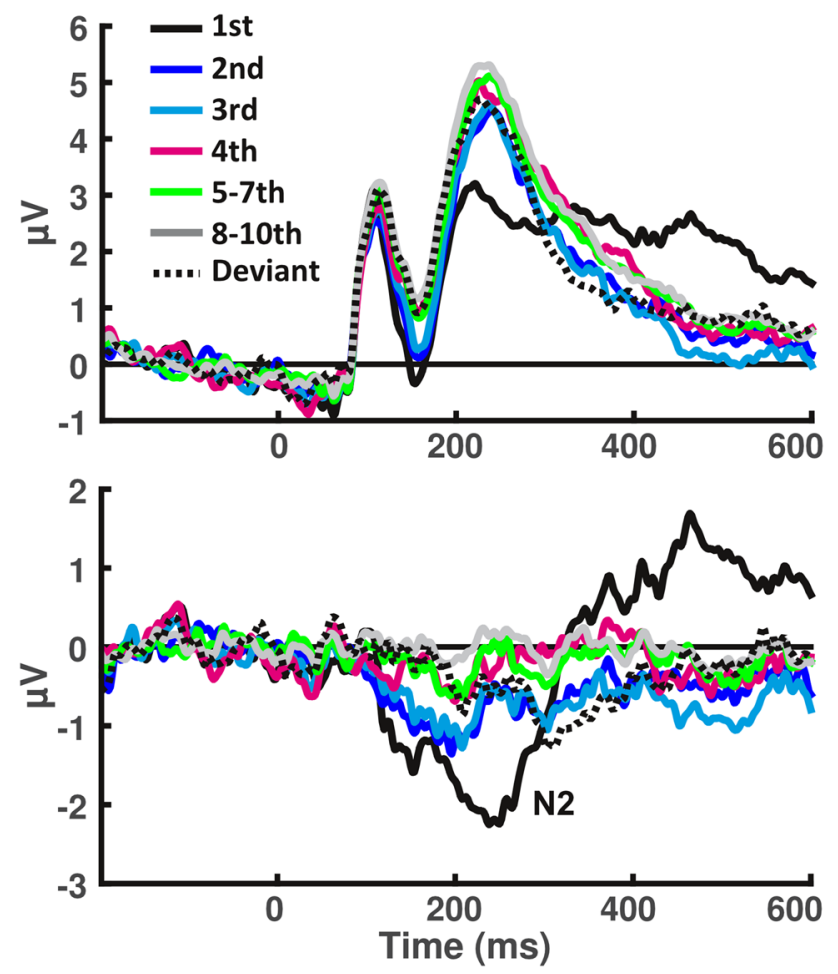

Fig. 4 Human ERP and difference waves from the parietal electrode, showing N2 used for analysis. Top: original human grand average ERPs of all conditions in electrode P2. Bottom: difference waves of all conditions for electrode $\mathrm{P} 2$ in humans

to temporo-occipital regions, the retrosplenial area, and the anterior cingulate. We also found an extended positivity in parietal electrodes from roughly 200 to $500 \mathrm{~ms}$, that we tentatively labeled P3 (Fig. 6d), more due to its position within the ERP-component sequence, rather than as a suggestion of it being analogous to the human P300.

\section{Repetition effects in human subjects are mostly linear and the deviant response occurs during the N2}

The P1 component (Fig. 7a) showed a significant repetition effect, $F(5,130)=5.32, p=0.001$. The linear, quadratic and cubic functions applied to the amplitudes of each time point averaged over subjects yielded $R_{\text {adj }}^{2}$ of $0.66,0.66$, and 0.79 , respectively. However, the Wilcoxon signed rank test did not indicate a significant difference when comparing these parameters derived from fitting each subjects time-amplitude data individually, $z=-0.192, p=0.84$, and $z=0.096$, $p=0.923$, for the comparisons of linear against quadratic and cubic fits, respectively. The effect of repetition was thus more of a linear nature. There was no significant effect of deviant stimuli on the P1 component, $t(26)=-1.02$, $p=0.32$.

For the N1 (Fig. 7a), we found a significant amplitude reduction over the repetition sequence, $F(5,130)=5.45$, $p=0.003$. The linear function was not significantly worse than the quadratic or cubic functions at explaining the data with $R_{\text {adj }}^{2}=0.691,0.955$ and 0.951 , respectively, $z=0.985$, $p=0.325, z=-1.87, p=0.061$. Again, the deviant effect was found to be insignificant $t(26)=0.32, p=0.74$.

The P2 (Fig. 7a) was also found to display a significant effect of repetition, $F(5,130)=9.06, p<0.001$, that was deemed to be following a quadratic function, with an average $R_{\text {adj }}^{2}=0.498,0.914$, and 0.983 for the linear, quadratic, and cubic functions applied on the averaged data, and $z=-2.306, p=0.021, z=-1.393, p=0.164$ for the comparisons between linear, and quadratic or cubic fits, respectively. Based on our increased $\alpha$ level, the deviant effect was non-significant, $t(26)=-2.1, p=0.045$.

The N2 (Fig. 7a), for which we conducted all analyses omitting the tenth presentation that instead served as a baseline to produce the difference waves, also showed a significant effect of repetition, $F(5,130)=10.49, p>0.001$. With an $R_{\text {adj }}^{2}=0.454,0.763$, and 0.842 , on the grand average, the quadratic function proved to be a better fit, compared to the linear function, with $z=-2.09$, and $p=0.037$, while the cubic function did not, $z=-1.345, p=0.179$. Furthermore, this difference wave showed a significant deviant effect, with the deviant condition producing a difference amplitude of -2.32 (SD: 2.11), compared to 0.96 (SD 1.4) found as an average of the eighth and ninth standard presentations, $t(26)=-2.78, p=0.01$.

Finally, the P300 (Fig. 7a) showed a strong novelty response, which lead to a significant repetition effect, $F(5$, $130)=20.54, p<0.001$. In contrast to all the previous components, this effect was found to be better explained by both the quadratic and cubic functions, rather than the linear function, with $z=-2.619$ and $p=0.009$ (for the quadratic comparison) and $z=-2.955$ and $p=0.003$ (for the cubic
Table 1 List of human ERPcomponents: Component-labels, labels of electrodes (according to the $10 \%$ extension of the international 10-20 system) and latencies used for analysis, as well as the average (Av.) amplitude and the nature of the repetition (Rep.) effect

\begin{tabular}{lllrl}
\hline Label & Electrodes & Latency $(\mathrm{ms})$ & \multicolumn{1}{c}{$\begin{array}{l}\text { Av. ampli- } \\
\text { tude }(\mu \mathrm{V})\end{array}$} & Rep. effect \\
\hline P1 & $P O 7, P O 3, P O z, P O 4, P O 8, O 1, O z, O 2$ & 110 & $6.91(3.64)$ & Linear \\
N1 & $P 5, P 3, P 1, P z, P 2, P 4, P 6$ & 150 & $0.7(2.95)$ & Linear \\
P2 & $P O 7, P O 3, P O z, P O 4, P O 8, O 1, O z, O 2$ & 240 & $10.76(4.95)$ & Quadratic \\
N2 & $P 2($ difference wave $)$ & $270-290$ & $-0.27(3.46)$ & Quadratic \\
P300 & $C P 3, C P 1, C P z, C P 2, C P 4, P z$ & $300-500$ & $1.69(1.54)$ & Quadratic \\
\hline
\end{tabular}




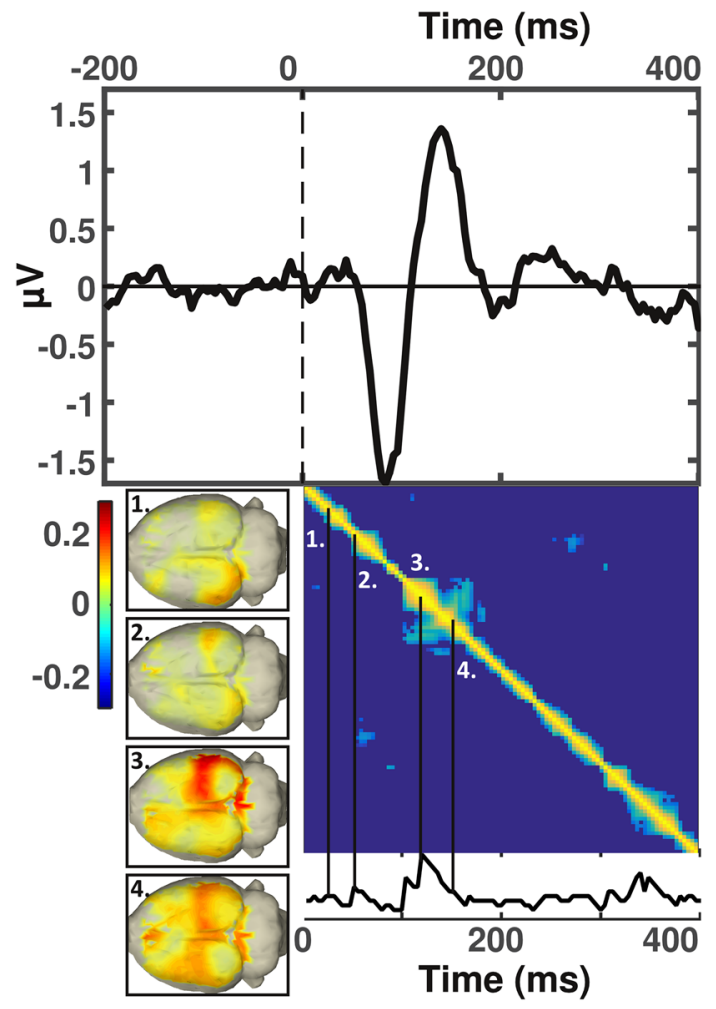

Fig. 5 Rodent event-related potential sources elicited by visuospatial information processing. Top: rodent grand average event-related potential (ERP) derived from posterior electrodes (situated above V1). Bottom right: representational similarity matrix calculated for the time span of $0-400 \mathrm{~ms}$ and with the significance level at at $p<0.05$ that includes a time series indicating across how many time points source-patterns correlated significantly. Bottom left: source estimates for time windows of interest corresponding to the numbering included in the bottom right panel

comparison). Comparing the quadratic and cubic functions among each other yielded no significant differences, $z=-1.634, p=0.102$. The respective goodness-of-fit on the subject averaged data was $R_{\text {adj }}^{2}=0.107,0.399$, and 0.666 for the linear, quadratic, and cubic functions, respectively. The deviant effect did not reach our significance threshold, with $t(26)=-2.37, p=0.025$.

In summary, for the human components, we consistently found significant effects of repetition, that were linear up until the P2, where a quadratic polynomial function was a better fit, as was the case for the $\mathrm{N} 2$ and $\mathrm{P} 300$ components. Deviant effects, in turn, were only found in the N2 difference wave.

\section{Repetition effects in the rodent are mostly non-linear, while the $\mathrm{N} 160$ is sensitive to deviance}

The rodent P40 (Fig. 7b) was not significantly altered by the repetition of the standard stimuli, or the presentation of a deviant, $F(5,60)=0.41, p=0.73$, and $t(12)=0.67, p=0.51$, respectively.

The N80 in turn showed a significant main-effect of repetition, $F(5,60)=3.85, p=0.018$ (Fig. $7 b$; Table 2). This effect showed a trend towards being better explained by the cubic function, with $z=-1.922$, and $p=0.055$, whereas the quadratic function did not yield a comparable decrease in error, $z=0.035, p=0.972$. The deviant effect was insignificant $t(12)=-1.12, p=0.28$.

The P135 showed a strong repetition effect, with $F(5$, $60)=11.83, p<0.001$, that was best explained by the cubic function $z=-2.83, p=0.005$ (Fig. 7b). With $R_{\text {adj }}^{2}=0.188$, 0.514 , and 0.657 , for average data fitted with linear, quadratic, and cubic functions, respectively, the difference between the quadratic and linear goodness-of-fit was not significantly different, $z=-1.433, p=0.152$. No significant deviant effect was found, $t(12)=-0.4, p=0.7$.

The N160, that we found restricted to the anterior cingulate electrode (Fig. 6g), also displayed a very strong novelty response, in line with a significant effect of repetition, $F(5,60)=17.05, p<0.001$ (Fig. 7b). Again this rodent component, with a linear fit of $R_{\text {adj }}^{2}=0.156$, a quadratic fit of $R_{\text {adj }}^{2}=0.514$ and a cubic fit of $R_{\text {adj }}^{2}=0.592$, was significantly non-linear over repeated presentation of standards, with $z=-2.062, p=0.039$ for the linear-quadratic comparison and $z=-2.201, p=0.028$ for the linear-cubic comparison. Quadratic and cubic functions did not differ from one another $z=1.013, p=0.311$, suggesting that this component was more aligned with the human components $\mathrm{P} 2$ or $\mathrm{N} 2$ in its behavior across repetitions. Furthermore, a variant of this component in the form of a slower and slightly later onset was found to be significantly responsive to deviants, based on a $t$ test on its mean amplitude $t(12)=-3.92, p=0.002$.

Finally, as the parietal P3 (Fig. 6d) extended across approximately $140 \mathrm{~ms}$ from 220 to $360 \mathrm{~ms}$, we used the largest positive amplitude in the two parietal electrodes during this time window for comparison. This component displayed a strong repetition effect, $F(5,60)=6.26, p<0.001$ (Fig. 7b). However, despite progressing very similarly to the human P300, this component turned out to be not significantly better explained by the non-linear function, which produced a fit of $R_{\text {adj }}^{2}=0.488$ (for the quadratic function) and $R_{\text {adj }}^{2}=0.496$ (for the cubic function), as opposed to the linear function that in fact displayed a negative $R^{2}$ adj of -0.066 , when fitting these curves to the subject average data points. When comparing the fits of linear and the two non-linear functions across subjects, the Wilcoxon test reported $z=-1.153, p=0.249$, and $z=-0.384, p=0.7$ for the comparison between linear and quadratic/cubic functions. Furthermore, the deviant effect was not significant, $t(12)=-1.11, p=0.29$. This may in part be due to the recovery of amplitudes in the ERPs of the ninth and tenth 
Fig. 6 Rodent visual eventrelated potentials respond to novelty. a-e Rodent grand average ERPs averaged over lateral electrodes across all conditions are shown (note that for $\mathbf{c}$ we only display the left electrode); $\mathbf{f}-\mathbf{g}$ grand average ERPs from the midline electrodes; $\mathbf{h}$ electrode layout. Green arrow indicates Bregma (overlaid by vertical blue line), red arrow indicates the midline (overlaid by horizontal blue line). $C g 1$ cingulate cortex area $1, \mathrm{FrA}$ frontal association area, $\mathrm{FrAz}$ central frontal association area, $G$ ground, $M 1$ primary motor cortex, PtA parietal association area, $S 1$ primary somatosensory cortex, V1M: primary visual cortex, monocular area, $I$ N160 in Cg1-electrode, $J \mathrm{P} 40, \mathrm{~N} 80$, and $\mathrm{P} 135$ in occipital electrodes
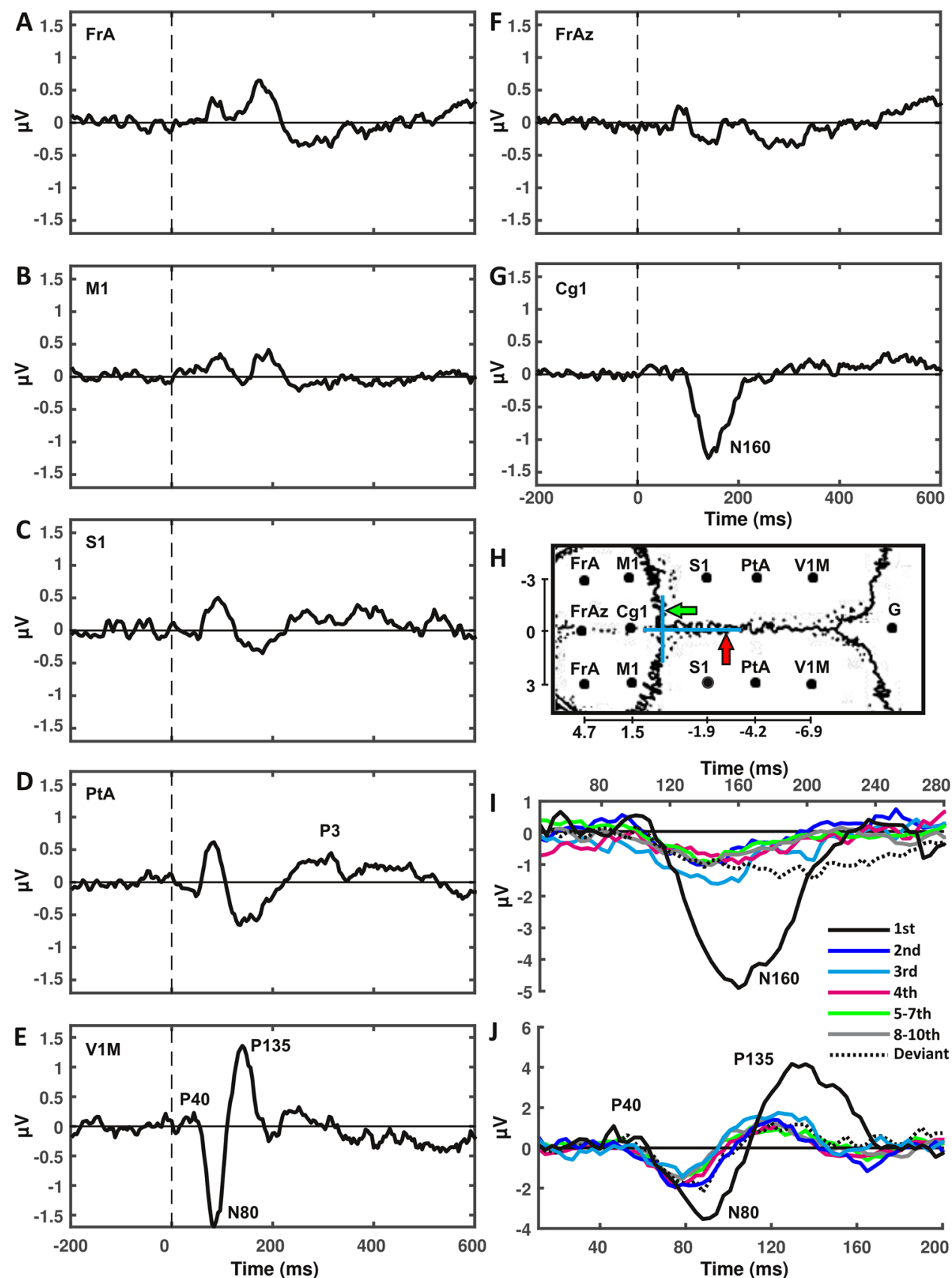

presentations in this component, or the aforementioned variance across our rodent cohort, both in terms of neural generators for the P3, as well as time.

To summarize, the early rodent components that were sensitive to repetition responded in accordance with a cubic polynomial function, whereas the human cohort displayed almost the opposite behavior, in that all components up until the P300 responded according to a linear or quadratic function. One striking commonality, however, is that both species responded to deviants in a late negativity (the rodent N160, and human N2) that coincided with the descending phase of the second positive deflection (the rodent $\mathrm{P} 135$ and human $\mathrm{P} 2$ ). The source estimates for this time window in both species appeared to involve cingulate, and potentially retrosplenial, areas. 


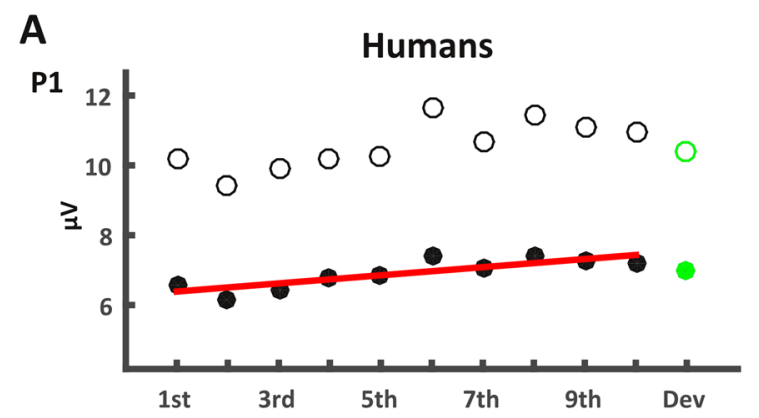

B
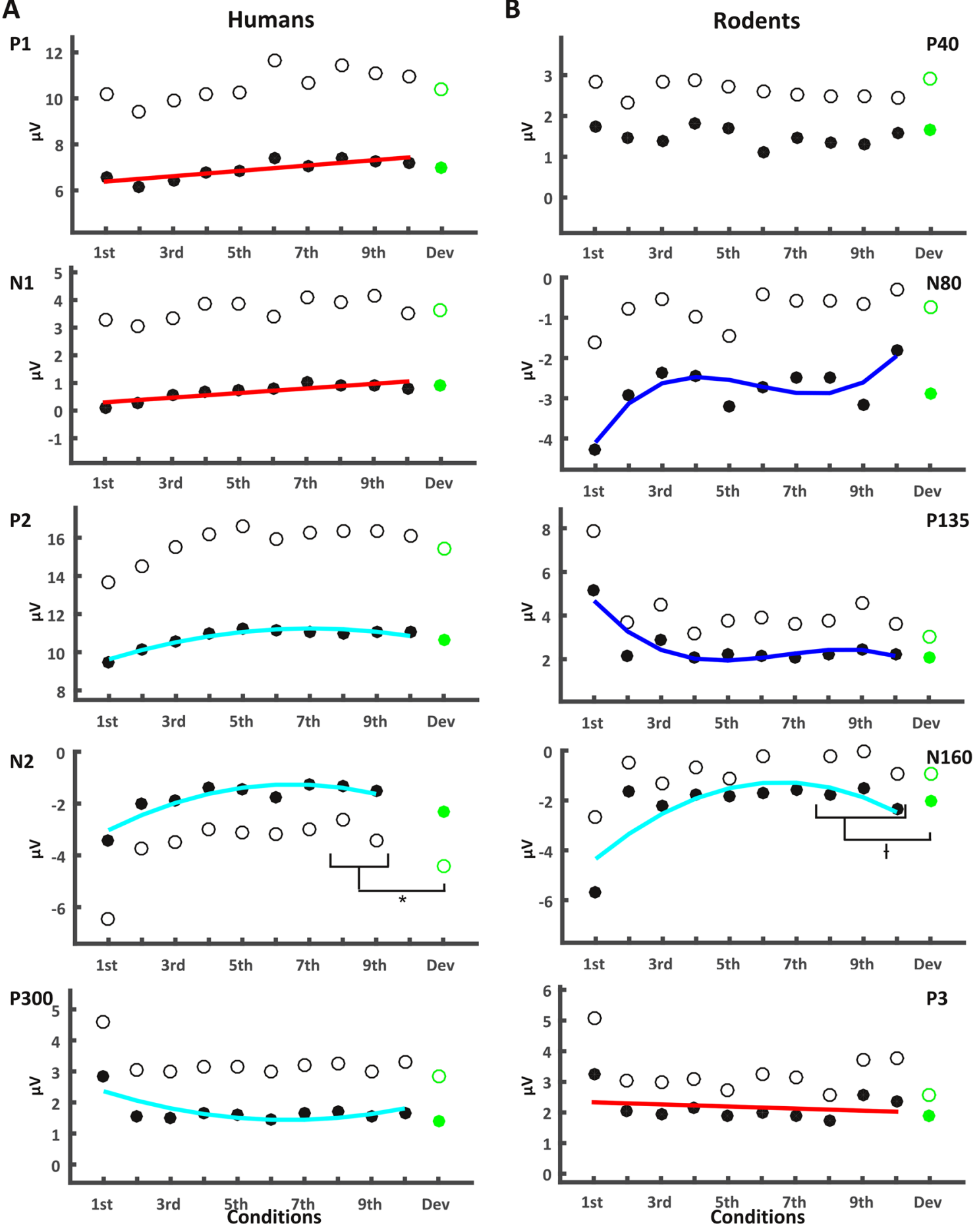

Fig. 7 Human repetition effects are mostly linear, rodent effects are mostly non-linear. Amplitudes for the repetitions (black dots, empty dots indicating standard deviation) and the deviant (green) different ERP-components, arranged in sequence of their occurrence for humans (left) and rodents (right). Red, cyan, and blue lines correspond to the linear, quadratic, or cubic polynomial functions fitted to

the subject-averaged amplitudes, respectively, depending on which function explained the effects best. An asterisk indicates a significant difference between the mean amplitude of the late repetition trials the deviant. A cross indicates a significant deviant effect for this component, corresponding to a slightly later time window 
Table 2 List of rodent ERP components: Their assigned labels, which electrodes and latencies were used for analysis, as well as their average amplitude and nature of the repetition effect, if present

\begin{tabular}{|c|c|c|c|c|}
\hline Label & Electrodes & Latency (ms) & $\begin{array}{l}\text { Av. ampli- } \\
\text { tude }(\mu \mathrm{V})\end{array}$ & $\begin{array}{l}\text { Repetition } \\
\text { effect }\end{array}$ \\
\hline P40 & $\begin{array}{c}V 1 M(\mathrm{~L} . \\
\& \mathrm{R} .)\end{array}$ & 40 & $2.42(3.28)$ & NS \\
\hline N80 & $\begin{array}{c}V 1 M(\mathrm{~L} . \\
\& \mathrm{R} .)\end{array}$ & 80 & $-1.15(3.26)$ & Cubic (trend) \\
\hline P135 & $\begin{array}{c}V 1 M(\mathrm{~L} . \\
\& \mathrm{R} .)\end{array}$ & 134 & $2.9(3.33)$ & Cubic \\
\hline N160 & $C G 1$ & $270-290$ & $-0.85(2.98)$ & Quadratic \\
\hline P3 & $P t A$ (L. \& R.) & $300-400$ & $2.63(3.23)$ & Linear \\
\hline
\end{tabular}

\section{Discussion}

The present study compared the cortical dynamics involved in object perception in rats and humans. This was done by engaging both species in an equivalent visuospatial task that involved showing them images of abstract, yet real, objects that were selected to minimize affordance characteristics. Both species viewed the images in an oddball-like paradigm, where objects were repeated up to 21 times, including one subtle configurational deviant, before a different object was shown in the same manner.

We found that source estimation of the novelty response in humans conforms with the hypothesized activation of ventral pathway regions, such as the inferotemporal cortex, that is central to object perception. This corresponded to the N1 component, that has, in previous studies, been linked to discrimination tasks (Vogel and Luck 2000; Hopf et al. 2002), although usually dependent on attention. Perhaps the most pronounced effect identified by our source estimates was the strong increase in the area of the posterior midline, near the cingulate, or retrosplenial areas, in the time window of the P2 and N2. Interestingly, this was also the only component sensitive to the deviant stimulus.

The source estimates of the rodents displayed a comparable progression, in that visual cortex activity at around $40 \mathrm{~ms}$ was followed by a very rapid spread of increased source power from the parietal to lateral occipital and retrosplenial areas, from $80 \mathrm{~ms}$ to roughly $150 \mathrm{~ms}$ after stimulus presentation. The eccentricities that we found in the ERPs corresponded well to the stages that were separable from one another in the multivariate correlation analysis. More importantly, together with the source estimates, the first ERPcomponents appear to fit neatly to those identified using single-unit recordings by Vermaercke et al. (2014). They reported neuronal responses in visual cortices at a latency of $40 \mathrm{~ms}$, followed by lateral areas at around $80 \mathrm{~ms}$. This would suggest that the spread from primary to lateral visual areas is indeed representative of complex object processing.
Taking into account an overall shorter response latency in the rodent, this may correspond to the occipital and inferior temporal increase in source power observed during the $\mathrm{P} 1$ and $\mathrm{N} 1$ in humans. One difference in the progression of novelty responses that we identified in rodents and humans is that in the rat, the parietal area became involved in novelty processing at an earlier stage than seen in humans. This contrasts to reports from primate studies that describe that the parietal cortex, as a part of the dorsal stream, fulfills an action-centered role (Goodale et al. 1991; Goodale and Milner 1992).

We would like to speculate that the rodent P135 and N160 correspond to the human P2 and N2. However, in our human subjects, the $\mathrm{P} 2$ and $\mathrm{N} 2$ were difficult to disambiguate on a temporal and topographical level, and the rodent P135 and N160 also appeared to form a complex. Thus this question remains unanswered. Nonetheless, both the human P2/N2 and the rodent P135/N160 were responsive to deviants, and appeared to both involve posterior cingulate, or retrosplenial, areas. Possibly these complexes of the N2/P2 and P135/ N160 may reflect a higher order mismatch detection that may be comparable between the species (Czigler et al. 2006; Winkler and Czigler 2012; Stefanics et al. 2014).

How do the early rodent ERP-components we observed in our visuospatial paradigm compare to previous studies of rodent ERPs? Most other studies, especially those that targeted more complex processing streams in the context of comparative investigations of human and rodent ERPs, have largely been restricted to the auditory modality (e.g., Brankačk et al. 1996; Ehlers et al. 1994; Hurlbut 1987; Meeren et al. 2001; Nguyen and Lin 2014; Sambeth and Maes 2006; Sambeth et al. 2003, 2004). Some studies have identified a number of different components that appear within roughly $50 \mathrm{~ms}$ after stimulus onset (de Bruin et al. 2001; Quian Quiroga and Van Luijtelaar 2002). Other studies reported components that extended over a longer period of $200 \mathrm{~ms}$ after stimulus onset (Sambeth et al. 2004; Sambeth and Maes 2006). Some of these components have been scrutinized in light of stimulus repetition. In a study that directly compared rats and humans, Sambeth et al. (2004) presented task-irrelevant tones and found that both species tended to have a similar progression of components, albeit at different latencies. These were labeled as P30, N70, P115, and N195, and considered as potential analogs of the human $\mathrm{P} 1, \mathrm{~N} 1, \mathrm{P} 2$, and N2, respectively. A cross-species commonality of showing repetition effects was found for the N70/ $\mathrm{N} 1$, the P115/P2, and the N195/N2, although the magnitude of those effects was different across species (Sambeth et al. 2004).

Finally, the question as to why both of our cohorts failed to show the expected deviance effect at the level of the P300/P3 remains. Given that in humans, the P300 is believed to reflect updating processes (Polich 2007), one 
potential explanation is that for our human cohort the deviant was too subtle to initiate this response. While this is purely speculative, we may still ask whether the rodent P3 is a candidate for a visually evoked P300. For the novelty response, the human $\mathrm{P} 300$ appeared to be mostly frontally distributed, whereas the rodent equivalent did not localize to a particular source, which may be due to the fact that it may arise from subcortical structures (Knight 1996). Furthermore, the human P300 and the rodent P3 were characterized by different electrophysiological profiles, although overall, their progressions over repetitions appeared to be almost identical (Fig. 7). However, given that neither human nor rodent showed the classical P300response to deviant stimuli warrants caution with regard to such interpretations.

A component potentially equivalent to the human P300 has repeatedly been reported in active auditory oddball paradigms performed by rats, where the animals were trained to respond to a particular deviant tone (Hurlbut 1987; Yamaguchi et al. 1993; Ehlers et al. 1994; Brankačk et al. 1996; Shinba 1997; Sambeth et al. 2003). These findings have been ambiguous with regard to the latency of this component, with some studies suggesting a proportionate latency to the preceding components, thus an earlier occurrence than in humans (Yamaguchi et al. 1993), and others reporting a more variable, potentially even later occurrence of the rodent $\mathrm{P} 3$ relative to the human equivalent (Sambeth et al. 2003). This may also have been a factor in the current study, in that there may be more variability in how and when the rodent P300-equivalent is elicited.

In conclusion, this study offers the first description and comparison of the broad topography of ERPs recorded from rats and humans during passive perception of complex and abstract objects. We found a similar progression of the novelty response in both species, whereas the response to object repetition differed between species. Interestingly, both species responded to deviant configurations of the objects in a similar way. These results suggest the ERPs evoked by passive visuospatial information processes exhibit a surprising degree of equivalency despite the vastly different cognitive capacities of the species studied. These findings suggest that ERPs generated in rats by novelty and deviance in this manner could serve as a basis for the study of the underlying neural, synaptic, and neurobiological mechanisms of the equivalent response in humans.

Acknowledgements This study was supported by the German Research Foundation (Deutsche Forschungsgemeinschaft, DFG) (SFB 874/ B3, project number: 122679504). We thank Nadine Kollosch for animal care.

Author contributions DM-V created the concept and strategy of the study; human studies were conducted by $\mathrm{MH}$ based on approaches devised by $\mathrm{CB}$; JC-K did the rodent electrode implantations and conducted rodent experiments together with $\mathrm{MH}$; data analysis was conducted by $\mathrm{MH}$ and DM-V; the paper was written by $\mathrm{MH}$ and $\mathrm{DM}-\mathrm{V}$, with contributions from all authors.

\section{Compliance with ethical standards}

Conflict of interest The authors declare that no conflicting interests exist.

Open Access This article is distributed under the terms of the Creative Commons Attribution 4.0 International License (http://creativeco mmons.org/licenses/by/4.0/), which permits unrestricted use, distribution, and reproduction in any medium, provided you give appropriate credit to the original author(s) and the source, provide a link to the Creative Commons license, and indicate if changes were made.

\section{References}

Bellebaum C, Tettamanti M, Marchetta E, Della Rosa P, Rizzo G, Daum I, Cappa SF (2013) Neural representations of unfamiliar objects are modulated by sensorimotor experience. Cortex 49:1110-1125

Brankačk J, Seidenbecher T, Müller-Gärtner HW (1996) Task-relevant late positive component in rats: Is it related to hippocampal theta rhythm? Hippocampus 6:475-482

Czigler I, Weisz J, Winkler I (2006) ERPs and deviance detection: visual mismatch negativity to repeated visual stimuli. Neurosci Lett 401:178-182

de Bruin NM, Ellenbroek B, van Schaijk WJ, van Luijtelaar EL, Cools AR, Coenen AM (2001) Sensory gating of auditory evoked potentials in rats: effects of repetitive stimulation and the interstimulus interval. Biol Psychol 55:195-213

Delorme A, Makeig S (2004) EEGLAB: an open source toolbox for analysis of single-trial EEG dynamics including independent component analysis. J Neurosci Methods 134:9-21

DiCarlo JJ, Zoccolan D, Rust NC (2012) How does the brain solve visual object recognition? Neuron 73:415-434

Ehlers CL, Kaneko WM, Robledo P, Lopez A (1994) Long latency event-related potentials in rats: effects of task and stimulus parameters. Neuroscience 62:161-175

Felleman DJ, Van Essen DC (1991) Distributed hierachical processing in the primate cerebral cortex. Cereb Cortex 1:1-47

Gallant JL, Braun J, Van Essen DC (1993) Selectivity for polar, hyperbolic, and Cartesian gratings in macaque visual cortex. Science 80-:259:100-103

Ghio M, Schulze P, Suchan B, Bellebaum C (2016) Neural representations of novel objects associated with olfactory experience. Behav Brain Res 308:143-151

Goodale MA, Milner AD (1992) Separate visual pathways for perception and action. Trends Neurosci 15:20-25

Goodale MA, Milner AD, Jakobson LS, Carey DP (1991) A neurological dissociation between perceiving objects and grasping them. Nature 349:154-156. https://doi.org/10.1038/349154a0

Gramfort A, Papadopoulo T, Olivi E, Clerc M (2010) OpenMEEG: opensource software for quasistatic bioelectromagnetics. Biomed Eng Online 9:45

Gross CG, Bender DB, Rocha-Miranda CE (1969) Visual receptive fields of neurons in the inferotemporal cortex of the monkey. Science 80 166:1303-1306

Hopf JM, Vogel E, Woodman G, Heinze HJ, Luck SJ (2002) Localizing visual discrimination processes in time and space. J Neurophysiol 88:2088-2095 
Hurlbut BJ (1987) Auditory elicitation of the evoked potential in the rat. Physiol Behav 39:483-487

Ito M, Komatsu H (2004) Representation of angles embedded within contour stimuli in area V2 of macaque monkeys. J Neurosci 24:3313-3324

Kemp A, Manahan-Vaughan D (2012) Passive spatial perception facilitates the expression of persistent hippocampal long-term depression. Cereb Cortex 22:1614-1621

Knight RT (1996) Contribution of human hippocampal region to novelty detection. Nature 383:256-259

Kybic J, Clerc M, Abboud T, Faugeras O, Keriven R, Papadopoulo $\mathrm{T}$ (2005) A common formalism for the integral formulations of the forward EEG problem. IEEE Trans Med Imaging 24:12-28

Meeren HKM, Van Cappellen van Walsum AM, Van Luijtelaar ELJM, Coenen AML (2001) Auditory evoked potentials from auditory cortex, medial geniculate nucleus, and inferior colliculus during sleep-wake states and spike-wave discharges in the WAG/Rij rat. Brain Res 898:321-331

Mishkin M, Ungerleider LG, Macko KA (1983) Object vision and spatial vision: two cortical pathways. Trends Neurosci 6:414-417

Nguyen DP, Lin SC (2014) A frontal cortex event-related potential driven by the basal forebrain. Elife 2014:1-17

Ohki K, Chung S, Ch YH, Kara P, Reid RC (2005) Functional imaging with cellular resolution reveals precise micro- architecture in visual cortex. Nature 433:597-603

Oostenveld R, Fries P, Maris E, Schoffelen JM (2011) FieldTrip: open source software for advanced analysis of MEG, EEG, and invasive electrophysiological data. Comput Intell Neurosci 2011:1-9

Perrett DI, Rolls ET, Caan W (1982) Visual neurones responsive to faces in the monkey temporal cortex. Exp Brain Res 47:329-342

Polich J (2007) Updating P300: an integrative theory of P3a and P3b. Clin Neurophysiol 118:2128-2148

Quian Quiroga R, Van Luijtelaar ELJM (2002) Habituation and sensitization in rat auditory evoked potentials: a single-trial analysis with wavelet denoising. Int J Psychophysiol 43:141-153

Rüther NN, Clayton E, Klepp A, Bellebaum C (2014a) Observed manipulation of novel tools leads to mu rhythm suppression over sensory-motor cortices. Behav Brain Res 261:328-335

Rüther NN, Tettamanti M, Cappa SF, Bellebaum C (2014b) Observed manipulation enhances left fronto-parietal activations in the processing of unfamiliar tools. PLoS One 9:e99401

Sambeth A, Maes JHR (2006) A comparison of event-related potentials of humans and rats elicited by a serial feature-positive discrimination task. Learn Motiv 37:269-288
Sambeth A, Maes JHR, Van Luijtelaar G, Molenkamp IBS, Jongsma MLA, Van Rijn CM (2003) Auditory event-related potentials in humans and rats: effects of task manipulation. Psychophysiology 40:60-68

Sambeth A, Maes JHR, Quiroga RQ, Coenen AML (2004) Effects of stimulus repetitions on the event-related potential of humans and rats. Int J Psychophysiol 53:197-205

Shinba T (1997) Event-related potentials of the rat during active and passive auditory oddball paradigms. Electroencephalogr Clin Neurophysiol 104:447-452

Stefanics G, Kremláček J, Czigler I (2014) Visual mismatch negativity: a predictive coding view. Front Hum Neurosci 8:1-19

Tafazoli S, Safaai H, De Franceschi G, Rosselli FB, Vanzella W, Riggi M, Buffolo F, Panzeri S, Zoccolan D (2017) Emergence of transformation-tolerant representations of visual objects in rat lateral extrastriate cortex. Elife 6:1-39

Ungerleider LG, Mishkin M (1982) Two Cortical Visual Streams. In: Ingle DJ,, Goodale MA, Mansfield RJW (eds) Analysis of visual behavior. The MIT Press, Cambridge, pp 549-586

Valdés-Hernández PA, Sumiyoshi A, Nonaka H, Haga R, AubertVásquez E, Ogawa T, Iturria-Medina Y, Riera JJ, Kawashima R (2011) An in vivo MRI template set for morphometry, tissue segmentation, and fMRI localization in rats. Front Neuroinform $5: 1-19$

Vermaercke B, Gerich FJ, Ytebrouck E, Arckens L, Op de Beeck HP, Van den Bergh G (2014) Functional specialization in rat occipital and temporal visual cortex. J Neurophysiol 112:1963-1983

Vogel EK, Luck SJ (2000) The visual N1 component as an index of a discrimination process. Psychophysiology 37:190-203

Winkler I, Czigler I (2012) Evidence from auditory and visual eventrelated potential (ERP) studies of deviance detection (MMN and vMMN) linking predictive coding theories and perceptual object representations. Int J Psychophysiol 83:132-143

Yamaguchi S, Globus H, Knight RT (1993) P3-like potential in rats. Electroencephalogr Clin Neurophysiol Evoked Potentials $88: 151-154$

Publisher's Note Springer Nature remains neutral with regard to jurisdictional claims in published maps and institutional affiliations. 